\title{
$\Pi_{1}^{1}$ SETS OF UNBOUNDED LOEB MEASURE
}

\author{
BOŠKO ŽIVALJEVIĆ \\ (Communicated by Andreas R. Blass)
}

\begin{abstract}
For every $\Pi_{1}^{1}$ and non-Borel subset $P$ of an internal set $X$ in a $\aleph_{2}$ saturated nonstandard universe there exists an internal, unbounded, nonatomic measure $\mu$ so that $L(\mu)(P \triangle B)$ is not finite for any Borel set $B$ in $X$.
\end{abstract}

Let $\mathcal{H}=(X, \mu, \mathcal{A})$ be an internal measure space, i.e., $X$ is an internal set, $\mathcal{A}$ is an internal algebra of internal subsets of $X$ (i.e., $\mathcal{A}$ is closed with respect to taking finite unions and intersections and to taking complements) and $\mu$ is an internal finitely additive measure defined on $\mathcal{A}$. We can associate to $\mathcal{H}$ a standard, complete measure space $L(\mathcal{H})=(X, L(\mu), L(\mathcal{A}))$ by following the procedure of Loeb (for details see [Lo] or [StBa]). We obtain a $\sigma$ additive measure $L(\mu)$ defined on a complete $\sigma$ algebra of sets $L(\mathcal{A})$. The elements of $L(\mathcal{A})$ are usually called Loeb measurable sets. If the measure of the ground space $X$ is finite $L(\mathcal{H})$ is called bounded Loeb space and otherwise unbounded.

It is well known that, in the bounded measure case, for every Loeb measurable set $M$ in $X$ there exists a Borel (in fact $\Pi_{1}^{0}$ or $\Sigma_{1}^{0}$ ) set $B$ with $L(\mu)(P \triangle B)=0$. A natural question is, as posed by Chris Impens on the Galaxy news group (an electronic news group for Nonstandard Analysis), whether the above approximation holds not only in bounded but in unbounded Loeb spaces as well.

Question. Given an unbounded, Loeb measure space $L(\mathcal{H})$ and a Loeb measurable set $M$ of infinite measure in it, does there always exist a Borel set $B$ such that $L(\mu)(M \triangle B)=0$ ?

We shall give a negative answer to the above question by proving, in fact, that any $\Pi_{1}^{1}$ and non-Borel set $P$ can be made to be at nonfinite distance (in the sense of measure $L(\mu))$ from any Borel set $B$ for some unbounded measure Loeb measure $L(\mu)$. This gives, from the point of view of Descriptive Set Theory of Internal Sets, the strongest possible counterexample to the Chris Impens question. The idea of the proof comes from the well-known construction of a nontrivial universally measure 0 set (a set in the unit interval $[0,1]$ of cardinality $\omega_{1}$ having $m$ measure 0 for every nonatomic Borel measure $m$ in $[0,1]$; see [Mi] for example).

First we give some definitions. The Borel hierarchy of sets over $\mathcal{A}$ is defined as usual. Elements of $\mathcal{A}$ are called $\Pi_{0}^{0}$ or $\Sigma_{0}^{0}$ sets. If we have defined the classes of $\Pi_{\beta}^{0}$ and $\Sigma_{\beta}^{0}$ sets for every $\beta<\alpha$, then we say that a set belongs to the class $\Pi_{\alpha}^{0}\left(\Sigma_{\alpha}^{0}\right)$ if

Received by the editors July 5, 1994 and, in revised form, January 31, 1995.

1991 Mathematics Subject Classification. Primary 03H04, 03E15, 28E05; Secondary 04A15.

(C)1996 American Mathematical Society 
it is an intersection (union) of a countable sequence of sets, each member of which belongs to a class $\Pi_{\alpha}^{0}$ or $\Sigma_{\alpha}^{0}$ for some $\alpha<\beta$.

A set $S$ is $\Sigma_{1}^{1}$ over $\mathcal{A}$ if it can be represented as $\bigcup_{\xi \in \omega^{\omega}} \bigcap_{n \in \omega} A_{\xi \mid n}$ for some sequence $A_{s} \in \mathcal{A}\left(s \in \omega^{<\omega}\right)$. $P$ is $\Pi_{1}^{1}$ over $\mathcal{A}$ if its complement is $\Sigma_{1}^{1}$ over $\mathcal{A}$. Given an infinite cardinal $\kappa$, a set $S$ is $\Sigma_{1}^{0}(\kappa)\left(\Pi_{1}^{0}(\kappa)\right)$ over $\mathcal{A}$ if it is a union (intersection) of $\kappa$ many internal sets from $\mathcal{A}$. It is well known that $\Sigma_{1}^{1}, \Pi_{1}^{1}, \Sigma_{1}^{0}(\kappa)$ and $\Pi_{1}^{0}(\kappa)$ sets over $\mathcal{A}$ are always Loeb measurable for any bounded or unbounded Loeb measure $L(\mu)$, provided that the nonstandard universe is $\kappa^{+}$saturated.

A set $S$ is countably determined over $\mathcal{A}$ (or, sometimes, countably based) if it can be represented as $\bigcup_{\xi \in \mathcal{I}} \bigcap_{n \in \omega} A_{n}^{\xi(n)}$ for some subset $\mathcal{I} \subseteq 2^{\omega}$ and a sequence $A_{n} \in \mathcal{A}$ (here we define $A^{0}=A$ and $A^{1}=\Omega-A$ ). The class of Borel sets is contained in the classes of $\Pi_{1}^{1}$ and $\Sigma_{1}^{1}$ sets (in fact, it is equal to their intersection), and the class of countably determined sets contains both the Borel class and the projective class of finite rank.

We say that $S$ is Borel, $\Sigma_{1}^{1}, \Pi_{1}^{1}$ or countably determined (without referring to $\mathcal{A}$ ) if the algebra $\mathcal{A}$ is equal to the internal power set of $\Omega$.

A set $P$ is called a complete $\Pi_{1}^{1}$ set if it is $\Pi_{1}^{1}$ and not Borel. A complete $\Pi_{1}^{1}$ set can be obtained by pulling back via the standard part map any complete $\Pi_{1}^{1}$ subset of the unit interval. Another example of a complete $\Pi_{1}^{1}$ set in the context of the Descriptive Set Theory of Internal Sets is given in [Ži2], Proposition 8.

The crucial property of $\Pi_{1}^{1}$ sets that we are going to use in this paper is their canonical decomposition into Borel sets. Every $\Pi_{1}^{1}$ over $\mathcal{A}$ set $P$ can be canonically expressed (see Proposition 7, (iii) in [Ži2]) as an increasing union $B_{\alpha}\left(\alpha<\omega_{1}\right)$ of Borel over $\mathcal{A}$ sets with the following Boundness Principle property (also well known in the standard Descriptive Set Theory): for every $\Sigma_{1}^{1}$ subset $S$ of $P$ there exists an $\alpha<\omega_{1}$ such that $S \subseteq B_{\alpha}$. In particular, every Borel subset $B$ of $P$ is completely contained in some $B_{\alpha}$. Also, the sequence $B_{\alpha}$ is unbounded from above with respect to $\subseteq$ relation if and only if $P$ is complete $\Pi_{1}^{1}$.

We shall call the above sequence $B_{\alpha}\left(\alpha<\omega_{1}\right)$ a canonical decomposition of $P$ into Borel sets.

The reader is referred to two standard references $[\mathrm{He}]$ and $[\mathrm{KKLM}]$ for the treatment of Descriptive Set Theory of Hyperfinite Sets. For the introduction to Nonstandard Analysis the reader is referred to [HuLo] and for the treatment of unbounded Loeb measure to [StBa].

Given a Loeb measure $L(\mu)$, we first characterize those $\Pi_{1}^{1}$ over $\mathcal{A}$ sets $P$ which are at finite distance from Borel over $\mathcal{A}$ sets. The characterization is in terms of canonical decomposition of $P$ into Borel sets.

Theorem 1 ( $\aleph_{1}$ saturation). For a given Loeb measure $L(\mu)$ and a $\Pi_{1}^{1}$ over $\mathcal{A}$ set $P$ the following are equivalent.

a) There exist a canonical decomposition $B_{\alpha}\left(\alpha<\omega_{1}\right)$ of $P$ into Borel sets and an ordinal $\alpha<\omega_{1}$ such that $L(\mu)\left(P-B_{\alpha}\right)$ is finite.

b) For every canonical decomposition $C_{\alpha}\left(\alpha<\omega_{1}\right)$ of $P$ into Borel sets there exists an ordinal $\alpha<\omega_{1}$ such that $L(\mu)\left(P-C_{\alpha}\right)$ is finite.

c) There exists a Borel over $\mathcal{A}$ set $B$ with $L(\mu)(B \triangle P)$ finite.

Proof. The Boundness Principle for canonical decompositions implies that a) and b) are always equivalent. Also, a) implies c). We prove that c) implies b). Suppose that $L(\mu)(B \triangle P)$ is finite for some Borel over $\mathcal{A}$ set $B$ and that $C_{\alpha}\left(\alpha<\omega_{1}\right)$ is a 
canonical decomposition of $P$ into Borel over $\mathcal{A}$ sets. We have that $P \triangle M=B$ for some set $M$ of finite measure, i.e., $(P-M) \cup N=B$ for some $M$ and $N$ of finite measure. We can suppose that $N$ is Borel over $\mathcal{A}$ by enlarging it by a Borel over $\mathcal{A}$ superset of the same measure if necessary. Therefore, $P-(M \cup N)=B-N$ is a Borel subset of $P$. By Boundness Principle we have $P-(M \cup N) \subseteq C_{\alpha}$ for some $\alpha$. In other words, $P-B_{\alpha} \subseteq M \cup N$ so that $P-B_{\alpha}$ is of finite $L(\mu)$ measure.

We now give the answer for the question raised at the beginning of the paper.

Theorem 2 ( $\aleph_{2}$ saturation). Let $P$ be a $\Pi_{1}^{1}$ over $\mathcal{A}$ set. Suppose that $P$ is not Borel. Then, there exists an internal, nonatomic measure $\mu$ so that :

a) $L(\mu)(B \triangle P)$ is infinite for every Borel over $\mathcal{A}$ set $B$

b) there exists a $\Sigma_{1}^{0}\left(\omega_{1}\right)$ set $S \subseteq P$ so that $L(\mu)(B \triangle S)$ is infinite for every Borel over $\mathcal{A}$ set $B$.

Proof. Let $B_{\alpha}\left(\alpha<\omega_{1}\right)$ be a canonical decomposition of $P$ into Borel over $\mathcal{A}$ sets. We may assume that $B_{\alpha} \backslash \bigcup_{\beta<\alpha} B_{\beta}$ is uncountable for every $\alpha$. Indeed, the set of $\alpha$ 's for which this is not true cannot be cofinal in $\omega_{1}$ because if for some $\gamma$ we had $B_{\alpha} \backslash \bigcup_{\beta<\alpha} B_{\beta}$ countable for every $\alpha>\gamma$, then the set $P-B_{\gamma}$ would have cardinality $\aleph_{1}$. However, $P-B_{\beta}$ as a $\Pi_{1}^{1}$ (and this countably determined) uncountable set contains an infinite internal subset (Proposition 2.5 in [KKLM]). In turn, infinite internal sets in a $\aleph_{2}$-saturated universe have at least cardinality $\aleph_{2}-$ a contradiction.

For every $\alpha<\aleph_{1}$ choose an infinite internal set $A_{\alpha} \in \mathcal{A}$ in $B_{\alpha} \backslash \bigcup_{\beta<\alpha} B_{\beta}$ (possible by $\aleph_{1}$ saturation). By $\aleph_{2}$ saturation we can find a nonatomic internal measure $\mu$ with $\mu\left(A_{\alpha}\right)$ infinite for every $\alpha$.

Now a) follows from Theorem 1 because $P-B_{\alpha}$ is of infinite $L(\mu)$ measure for every $\alpha$.

For b) we prove that $\bigcup_{\alpha<\aleph_{1}} A_{\alpha}$ is at infinite $L(\mu)$ distance from any Borel over $\mathcal{A}$ set. Indeed, suppose that, in the same way as in the proof of Theorem 1 , $\left(\left(\bigcup_{\alpha<\aleph_{1}} A_{\alpha}\right)-M\right) \cup N=B$, where $B$ is Borel over $\mathcal{A}$ and $M$ and $N$ are of finite $L(\mu)$ measure. We may assume that $N$ is Borel. Taking away $N$ we get $\left(\bigcup_{\alpha<\aleph_{1}} A_{\alpha}\right)-(M \cup N)=B-N$ a Borel over $\mathcal{A}$ subset of $P$. By Boundness Principle we have $\left(\bigcup_{\alpha<\aleph_{1}} A_{\alpha}\right)-(M \cup N) \subseteq B_{\beta}$ for some $\beta$. In particular, $A_{\beta+1} \backslash(M \cup N) \subseteq$ $B_{\beta}$. Taking in account that $A_{\beta+1} \cap B_{\beta}=\emptyset$ we get $A_{\beta+1} \subseteq M \cup N$ which is impossible because we have chosen $\mu$ so that every $A_{\alpha}$ has an infinite measure - a contradiction.

Remark 3. Note that, using the identity $A \triangle B=A^{c} \triangle B^{c}, A^{c}$ being the complement of $A$, a set $A$ is at finite distance from a Borel set if and only if its complement is at finite distance from some Borel set. In particular, it follows from Theorem 2 that for every $\Sigma_{1}^{1}$ over $\mathcal{A}$ and non-Borel set $S$ there exists a nonatomic, unbounded Loeb measure $L(\mu)$ so that $S$ is at infinite measure distance from any Borel over $\mathcal{A}$ set $B$.

Remark 4. It is easy to show (again assuming $\aleph_{2}$ saturation) that the approximation of Borel sets from within or from outside by sets of lower Borel rank is also impossible in the unbounded Loeb measure case. There always exists a $\Pi_{2}^{0}$ set which cannot be approximated by $\Pi_{1}^{0}$ or $\Sigma_{1}^{0}$ sets in the above manner. Let $P$ and $L(\mu)$ be as in the proof of Theorem 2. For the sake of this example we assume that $X$ is a hyperfinite set and that $\mu$ is a uniformly distributed counting measure, 
i.e., $\mu$ is defined as $\mu(A)=|A| / H$ for some fixed hyperfinite integer $H$. The proof of Theorem 2 can be easily altered to achieve this. Let $Y$ be an infinite hyperfinite set and let $B$ be a $\Pi_{2}^{0}$ set in the product $X \times Y$ so that the first projection $\pi(B)$ of $B$ is equal to the complement $S$ of $P$ (every $\Sigma_{1}^{1}$ set is a projection of a $\Pi_{2}^{0}$ set as noticed by Henson). We define a measure $\nu$ for the internal subsets $\Gamma$ of $X \times Y$ by $\nu(\Gamma)=|\Gamma| / H$. It is clear that if $M \subseteq X \times Y$ and $L(\nu)(M)=0$, then $L(\mu)(\pi(M))=0$. Now, suppose that $B \subseteq M$ with $M$ a $\Pi_{1}^{0}$ set and $L(\nu)(M-B)=0$. Then, $L(\mu)(\pi(M)-\pi(B)) \leq L(\mu)(\pi(M-B))=0$. However, the projection of a $\Pi_{1}^{0}$ set is always a $\Pi_{1}^{0}$ set (assuming $\aleph_{1}$-saturation) and we obtain that $S$ is at distance zero from a Borel set $\pi(M)$ - a contradiction. Similarly for $\Sigma_{1}^{0}$ sets.

We now turn to the analogous question of approximating $\Pi_{1}^{1}$ and $\Sigma_{1}^{1}$ sets by Borel sets in the sense of category. In [KeLe] Keisler and Leth introduced and studied the notion of being $U$ nowhere dense and $U$ meager in the context of subsets of the initial segment of the nonstandard model of integers. Let us fix an initial segment $X=\{0,1,2, \ldots, H\}$ of the nonstandard version of integers. An initial segment $U$ of $X$ is a cut if it is closed with respect to addition. We write $x>U$ if $x>y$ for every $y \in U . M \subseteq X$ is called $U$ nowhere dense if every interval $I \subseteq X$ of length $>U$ contains an interval $J$ of length $>U$ so that $J \cap M=\emptyset$. A set is $U$ meager if it is a union of countably many $U$ nowhere dense sets. The cofinality $c f(U)$ and the coinitiality $\operatorname{ci}(U)$ of $U$ is defined as the cofinality and the coinitiality of $U$ and $X-U$ respectively. By analogy to standard terminology we say that a set $A$ possesses $U$ property of Baire if $A \triangle M=B$ for some Borel set $B$ and some $U$ meager set $M$.

The reader is referred to [KeLe] for the rest of the definitions and results concerning $U$ meager sets.

The next theorem is analogous to Theorem 1. The only difference in the proof is that we needed the category analog to the fact that any measure zero set is contained in a Borel set of measure zero. This analogue is provided, in the case of cuts of uncountable cofinalities and coinitialities, by a result from [Ži1].

Theorem 5 ( $\aleph_{1}$ saturation). Let $U$ be a cut in $X$ with $\min \{c f(U), c i(U)\} \geq \omega_{1}$. Let $P \subseteq X$ be $a \Pi_{1}^{1}$ set. Then, the following three statements are equivalent.

a) There exist a Borel set $B$ and a $U$ meager set $M$ with $P \triangle M=B$.

b) There exists a canonical decomposition $B_{\alpha}\left(\alpha<\omega_{1}\right)$ of $P$ into Borel sets such that for some $\alpha<\omega_{1} P-B_{\alpha}$ is $U$ meager.

c) For every canonical decomposition $C_{\alpha}\left(\alpha<\omega_{1}\right)$ of $P$ into Borel sets there exists $\alpha<\omega_{1}$ such that $P-C_{\alpha}$ is $U$ meager.

Proof. b) and c) are always equivalent and obviously b) implies a). Suppose that a) is true and let $C_{\alpha}\left(\alpha<\omega_{1}\right)$ be a canonical decomposition of $P$ into Borel sets. Then, as $P$ and $B$ are countably determined sets, $M$ must be countably determined as well. Thus, $N=M-P$ is also countably determined and $(P-M) \cup N=B$. $M$ and $N$ are both $U$ meager sets. By Proposition 1.2 in [Ži1], there exists a $\Sigma_{1}^{0} U$ meager set $Q$ containing $N$. We have $(P-M) \cup Q=B \cup Q$ and $P-(M \cup Q)=B-Q$. By the Boundness Principle we have $B-Q \subseteq C_{\alpha}$ for some $\alpha<\omega_{1}$. Therefore, $P-C_{\alpha} \subseteq M \cup Q$ and $P-C_{\alpha}$ is $U$ meager.

\section{FINAL REMARKS}

Let $X=\{0,1, \ldots, H\}$ as before, an initial segment of the nonstandard model of integers, and $U$ a cut in $X$ with $\min \{c f(U), c i(U)\} \geq \omega_{1}$. Let $K \in U$ be an infinite 
natural number and $\mu_{K}$ the counting measure associated with $K$, i.e., $\mu_{K}(A)=$ $|A| / K$ for every internal subset $A$ of $X$. H. J. Keisler pointed out (during the discussion on the Galaxy news group) that by Proposition $7.7 \mathrm{~d}$ ) in [KeLe] there exists an ultrapower of the set of natural numbers $\omega$ so that $U$ is not only Loeb measurable for every $L\left(\mu_{K}\right)$ but, even stronger, it meets every internal set of finite $L\left(\mu_{K}\right)$ measure in an internal set. We shall now prove, using the idea of the proof of Theorem 2, that $U$ cannot be approximated not only by any Borel set but by no countably determined set, as well.

Theorem 6. Let $U$ be as above and let $K \in U$ be a fixed infinite integer. Then, there do not exist a countably determined set $C$ and a set $M$ of finite $L\left(\mu_{K}\right)$ measure so that $U \triangle M=C$.

Proof. The proof will follow the same lines as the proof of Theorem 2 once we establish the following two facts similar to the properties of canonical decompositions of $\Pi_{1}^{1}$ sets that we have extensively used so far.

Fact i). Any countably determined set $B \subseteq U$ is contained in some interval $[0, K] \subseteq U$.

Proof. The requirement $\min \{c f(U), c i(U)\} \geq \omega_{1}$ is equivalent to saying that $U$ is not countably determined (see Proposition 5.5 in [KeLe]). Now, if $B$ is cofinal in $U$, then the predicate $x \in U$ could have been defined as $(\exists y)(x<y \wedge y \in B)$ which defines a countably determined set.

Fact ii). For any $L \in U$ the set $\{x \in U: x>L\}$ is of infinite $L\left(\mu_{K}\right)$ measure.

Proof. As $U$ is closed with respect to addition we have that $L+k \cdot K \in U$ for all standard integers $k$. Each of the intervals $[L+k \cdot K, L+(k+1) \cdot K]$ is of $L\left(\mu_{K}\right)$ measure 1 .

Now, suppose that $U \triangle M=C$ for some set $M$ of finite $L\left(\mu_{K}\right)$ measure and some countably determined set $C$. In other words we have $(U-M) \cup N=C$ for $M$ and $N$ of finite $L\left(\mu_{K}\right)$ measure. We can assuming that $N$ is Borel. We get $U-(M \cup N)=C-N \subseteq U$. By Fact i) we have $U-(M \cup N) \subseteq[0, L] \subseteq U$ for some $L \in U$. That is, $U-[0, L] \subseteq M \cup N$ which is impossible because by Fact ii) $U-[0, L]$ is of infinite $L\left(\mu_{K}\right)$ measure.

For arbitrary Loeb measure $L(\mu)$ we, in fact, have the following theorem, the proof of which is analogous to the proof of Theorem 6 above.

Theorem 7. Let $U$ be a cut with $\min \{c f(U), c i(U)\} \geq \omega_{1}$. Let $L(\mu)$ be the Loeb measure of an internal measure $\mu$. Then the following statements are equivalent.

a) $U \triangle M=C$ for some countably determined set $C$ and a set $M$ of finite $L(\mu)$ measure.

b) $U \triangle M=A$ for some internal set $A$ and a set $M$ of finite $L(\mu)$ measure.

c) There exists an $L \in U$ such that $U-[0, L]$ is of finite $L(\mu)$ measure.

Note. The conjecture that a cut $U$ with uncountable cofinality and coinitiality in some nonstandard universe is not a.e. equal to any Borel set for any $L\left(\mu_{K}\right)$ above is made by Martin Kalina.

After I announced my Theorem 1 in the Galaxy news group Vladimir Kanovei gave the following example of a $\Sigma_{1}^{1}$ complete set $\Gamma$ and an unbounded Loeb measure 
$L(\mu)$ so that $\Gamma$ is not at distance 0 from any Borel set $B$ (notice that being at distance 0 or at finite distance from a Borel set is equivalent). His example is a little simpler than the usage of canonical decomposition of complete $\Pi_{1}^{1}$ sets but, in turn, it does not prove that any complete $\Sigma_{1}^{1}$ set can be made to be at infinite distance form any Borel set as Theorem 1 did.

I thank Professor Kanovei for allowing me to reproduce his example in this paper.

Let $S \subseteq X$ be a complete $\Sigma_{1}^{1}$ subset of $X$, (i.e., $S$ is $\Sigma_{1}^{1}$ and non-Borel). Let $\Gamma \subseteq X \times X$ be defined as $(x, y) \in \Gamma$ if and only if $y \in S$. $\Gamma$ is obtained by translating a copy of $S$ into every vertical section in $X \times X$. It is clear that $\Gamma$ is $\Sigma_{1}^{1}$. Let $L(\mu)$ be any Loeb measure with the property that the measure of any internal subset of $X \times X$ of cardinality $H+1$ is 1 , where $H$ is an infinite but fixed hyperfinite integer. For example, $X$ can be chosen to be a hyperfinite set of cardinality $H$ and $\mu$ might be defined as $\mu(A)=|A| / H$ for all hyperfinite subsets $A$ of $X \times X$.

Claim. There does not exist a Borel set $B \subseteq X \times X$ so that $L(\mu)(\Gamma \triangle B)=0$.

Proof. (Kanovei) Suppose that the claim is not true. Let $L(\mu)(\Gamma \triangle B)=0$ for some Borel $B \subseteq X \times X$. Let $A \supseteq \Gamma \triangle B$ be an internal set with $L(\mu)(A)<1 / 2$. Then the cardinality of $A$ is strictly less than $H+1$. This in turn means, by cardinality argument, that for some $x \in X$ the vertical section $A(x)$ of $A$ and thus the vertical section $(\Gamma \triangle B)(x)$ of $\Gamma \triangle B$ at $x$ are empty. Therefore, we have $\emptyset=(\Gamma \triangle B)(x)=\Gamma(x) \triangle B(x)=S \triangle B(x)$. In other words $S=B(x)$ and $S$ is Borel-a contradiction.

\section{REFERENCES}

[He] C. W. Henson, Analytic Sets, Baire Sets, and the Standard Part Map, Canadian J. Math. 31 (1979), 663-672. MR 80i:28019

[HuLo] A. E. Hurd and P. A. Loeb, An Introduction to Nonstandard Real Analysis, Academic Press, New York, 1985. MR 87d:03184

[Ji] R. Jin, Cuts in hyperfinite time lines, J. of Symbolic Logic 57 (1992), 522-527. MR 93j:03029

[KKLM] H.J. Keisler, K. Kunen, A. Miller and S. Leth, Descriptive Set Theory over Hyperfinite sets, J. Symbolic Logic 54 (4) (1989), 1167-1180. MR 91c:03040

[KeLe] H.J. Keisler and S. Leth, Meager Sets on the Hyperfinite Time Line, J. Symbolic Logic 56 (1) (1991), 71-102. MR 93a:03074

[Lo] P. A. Loeb, Conversion from Nonstandard to Standard Measure Space and Applications in Probability Theory, Trans.Amer.Math.Soc. 211 (1975), 113-122. MR 52:10980

[Mi] A. Miller, Special Subsets of the Real Line, Handbook of Set-Theoretical Topology (K. Kunene and J.E. Vaughan (), eds.), North Holland, Amsterdam, 1984, pp. 201-234. MR 86i: 54037

[StBa] K. D. Stroyan and J. M. Bayod, Foundations of infinitesimal stochastic analysis, NorthHolland, Amsterdam, 1986. MR 87m:60001

[Ži1] B. Živaljević, $U$-meager sets when the cofinality and the coinitiality of $U$ are uncountable, J. of Symbolic Logic 56 (1991), 906-914. MR 92k:03033

[Ži2] _ Lusin-Sierpinski Index for the Internal Sets, J. of Symbolic Logic 57 (1992), 172-178. MR 94a:03096

Department of Computer Science, The University of Illinois at Urbana-Champaign, URBANA, ILLINOIS 61801

E-mail address: zivaljev@cs.uiuc.edu

Current address: Process Management Computer, International Paper, 3101 International Rd.

E., Mobile, Alabama 36616

E-mail address: BZIVALJE@ipaper.com 